\title{
Advancing Diagnostic Safety Research: Results of a Systematic Research Priority Setting Exercise
}

\author{
Laura Zwaan, $\mathrm{PhD}^{7}$ (D) , Robert El-Kareh, MD, MPH, MS², Ashley N. D. Meyer, PhD ${ }^{3,4}$, \\ Jacky Hooftman, $\mathrm{MSc}^{7}$, and Hardeep Singh, $\mathrm{MD}, \mathrm{MPH}^{3,4}$
}

${ }^{1}$ Erasmus Medical Center Rotterdam, Institute of Medical Education Research Rotterdam, Rotterdam, The Netherlands; ${ }^{2}$ Department of Medicine, University of California at San Diego, San Diego, CA, USA; ${ }^{3}$ Center for Innovations in Quality, Effectiveness and Safety, Michael E. DeBakey VA Medical Center, Houston, TX, USA; ${ }^{4}$ Baylor College of Medicine, Houston, TX, USA.

\begin{abstract}
BACKGROUND: Diagnostic errors are a major source of preventable harm but the science of reducing them remains underdeveloped.

OBJECTIVE: To identify and prioritize research questions to advance the field of diagnostic safety in the next 5 years. PARTICIPANTS: Ninety-seven researchers and 42 stakeholders were involved in the identification of the research priorities.
\end{abstract}

DESIGN: We used systematic prioritization methods based on the Child Health and Nutrition Research Initiative (CHNRI) methodology. We first invited a large international group of expert researchers in various disciplines to submit research questions while considering five prioritization criteria: (1) usefulness, (2) answerability, (3) effectiveness, (4) potential for translation, and (5) maximal potential for effect on diagnostic safety. After consolidation, these questions were prioritized at an in-person expert meeting in April 2019. Top-ranked questions were subsequently reprioritized through scoring on the five prioritization criteria using an online questionnaire. We also invited non-research stakeholders to assign weights to the five criteria and then used these weights to adjust the final prioritization score for each question.

KEY RESULTS: Of the 207 invited researchers, 97 researchers responded and 78 submitted 333 research questions which were then consolidated. Expert meeting participants $(n=21)$ discussed questions in different breakout sessions and prioritized 50, which were subsequently reduced to the top 20 using the online questionnaire. The top 20 questions addressed mostly system factors (e.g., implementation and evaluation of information technologies), teamwork factors (e.g., role of nurses and other health professionals in the diagnostic process), and strategies to engage patients in the diagnostic process.

CONCLUSIONS: Top research priorities for advancing diagnostic safety in the short-term include strengthening systems and teams and engaging patients to support diagnosis. High-priority areas identified using these systematic methods can inform an actionable research agenda for reducing preventable diagnostic harm.

KEY WORDS: diagnostic safety; patient safety; research priorities; medical error.

Prior Presentation Diagnostic Error in Medicine (DEM) conference, November 2019

Received August 10, 2020

Accepted December 9, 2020

Published online February 9, 2021
J Gen Intern Med 36(10):2943-51

DOI: $10.1007 / \mathrm{s} 11606-020-06428-3$

(C) The Author(s) 2021

\section{INTRODUCTION}

High-quality research is essential to accelerate quality and safety of healthcare. ${ }^{1}$ One emerging risk area is diagnostic error, with estimates that at least 1 in 20 adults will have a diagnostic error annually in the outpatient setting. ${ }^{2}$ In hospitals, diagnostic error could involve $0.7 \%$ of adult hospitalizations and $5.6 \%$ of medical 7-day readmissions and many result in serious patient harm. ${ }^{3-5}$ However, substantial research gaps remain that limit diagnostic error reduction. ${ }^{6-8}$ This is not surprising because the diagnostic process is inherently complex and involves decisionmaking under uncertain conditions and limited time. ${ }^{9-11}$

The science of reducing diagnostic error remains underdeveloped and requires newer approaches, especially because the current medical research funding largely adopts a diseasefocused approach, whereas the diagnostic process cuts across thousands of diseases. ${ }^{12,13}$ Potential research opportunities can be broadly classified into three areas: error epidemiology, contributory factors, and interventions. ${ }^{14}$ The National Academies of Sciences, Engineering, and Medicine's (NASEM) report Improving Diagnosis in Health Care $^{15}$ defined diagnostic error as the failure to (a) establish an accurate and timely explanation of the patient's health problem(s) or (b) communicate that explanation to the patient. It concluded that that there is an urgent need for research on the diagnostic process and diagnostic errors and called for "a coordinated federal research agenda, committed funding, and significant public-private collaborations to enhance research in this critical area." The NASEM report included a list of potential research areas but prioritization of research areas and development of a specific set of actionable research questions to create impact on practice and patient care was considered outside the scope of the report. Efforts are needed to inform a deeper understanding of how to reduce missed opportunities in diagnosis and achieve correct and timely diagnosis while maximizing patient experiences. These efforts include identifying the main failure points given that diagnosis evolves over time within a complex sociotechnical health system, ${ }^{16,17}$ and developing, 
implementing, and testing specific interventions in order to achieve diagnostic excellence. ${ }^{18}$

In the past decade, few researchers have embarked on projects to advance the scientific understanding of diagnostic error and even fewer are using multidisciplinary perspectives to address evidence gaps. ${ }^{14,}{ }^{19-22}$ Much of this research is fragmented with little assurance it focuses on the right questions. ${ }^{23}$ Additional insights are needed to identify the most urgent and impactful questions to promote research that is more actionable and reduces patient harm. Therefore, we conducted a systematic research priority-setting exercise to identify and prioritize research questions to advance the field of diagnostic safety.

\section{METHODS}

\section{Overview}

We used established systematic research prioritization methods based on the Child Health and Nutrition Research Initiative (CHNRI). ${ }^{24}$ The CHNRI is a systematic and wellestablished method to identify research priorities. ${ }^{25-27} \mathrm{We}$ invited an international group of expert researchers in several disciplines related to diagnostic error to submit research questions. The submitted questions were prioritized using predefined prioritization criteria ensuring a transparent and systematic priority-setting process to minimize potential bias.

\section{Scope and Prioritization Criteria}

As a first step, the core research group (LZ, REK, AM, HS) defined the project scope as topics that would advance the field of diagnostic safety in the next 3-5 years in order to reduce patient harm in the diagnosis process. We also selected and adapted the prioritization criteria for developing and evaluating the research questions from the entire list of criteria described in the CHNRI approach (see Text Box 1). ${ }^{28}$ Diagnostic safety was defined as the prevention of errors and adverse effects to patients associated with the diagnostic process. We used the NASEM conceptualization of the diagnostic process. ${ }^{15} \mathrm{We}$ limited the scope by deeming screening decisions of asymptomatic patients as outside the focus of the project. However, research questions related to diagnostic evaluation of abnormal screening results were within scope. Treatment decisions were only included if they were relevant to the diagnostic process.

Text Box 1. Prioritization criteria

- Usefulness: Given the quality of existing evidence, how likely is it that the proposed research will fill a critical gap in knowledge?

- Answerability: How likely is it that the objectives will be met in the next 3-5 years given the current state of science and the size of the gap in knowledge?

- Effectiveness: How likely is it that the research question will generate or improve effective solutions related to diagnostic safety?

- Potential for translation: How likely is it that the research will generate knowledge that will be translated into widespread solutions to improve diagnostic safety?

- Maximum potential for effect on diagnostic safety: How likely is the proposed research to lead to significant improvement of diagnostic safety or reduction of diagnostic harm at a societal level? 


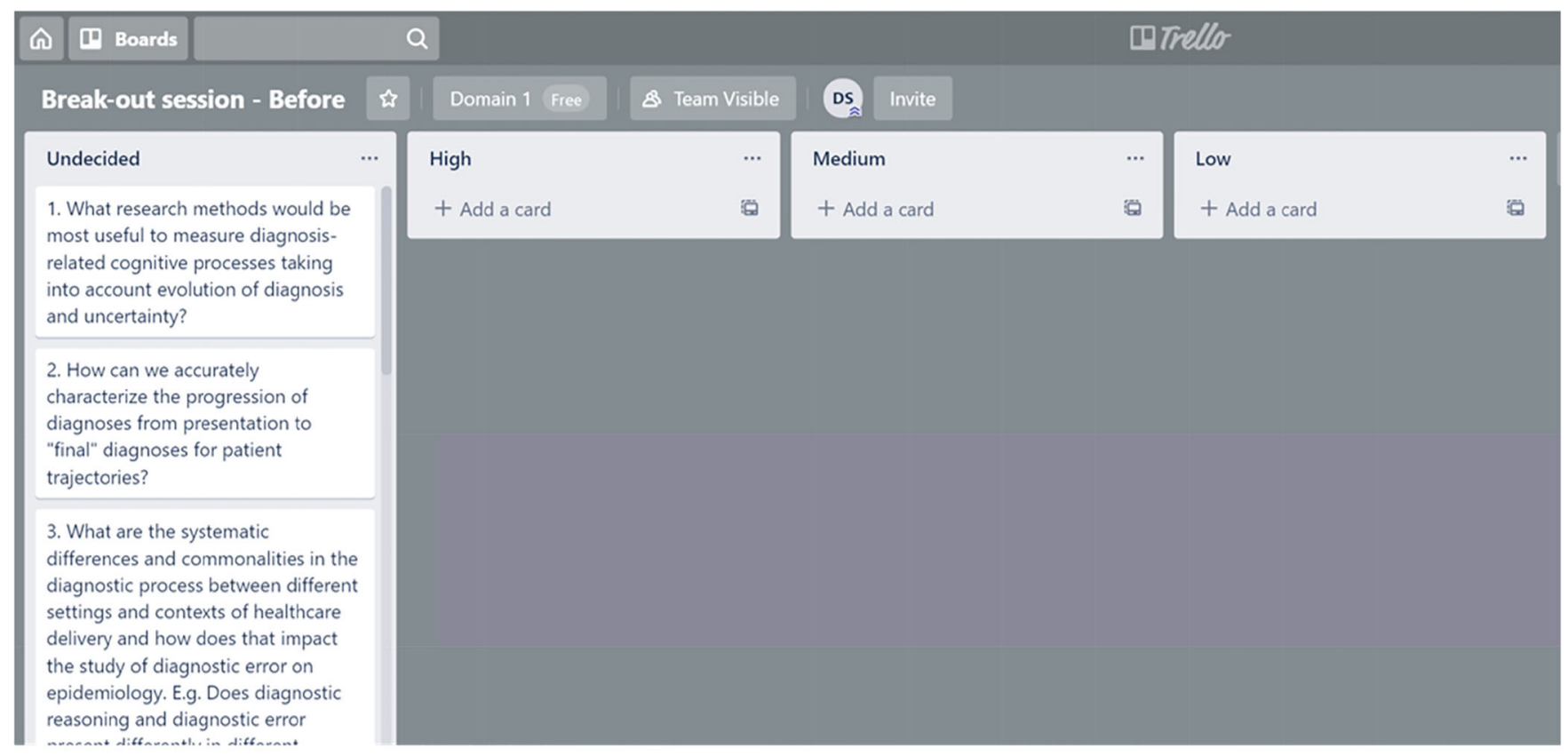

\begin{tabular}{|c|c|c|c|c|}
\hline A [1] Boards & \multicolumn{2}{|c|}{ Q } & \multicolumn{2}{|c|}{ पrello } \\
\hline Break-out session - After & 约 & Domain 1 Free & Invite & \\
\hline Undecided & $\cdots$ & High & Medium & Low \\
\hline \multirow[t]{4}{*}{ + Add a card } & \multirow[t]{4}{*}{ 缊 } & $\begin{array}{l}\text { 10. What definition of diagnostic } \\
\text { error informs the most useful } \\
\text { measurement for preventable } \\
\text { diagnostic harm? How can we define } \\
\text { a diagnostic error in a way that } \\
\text { acknowledges the unavoidable } \\
\text { uncertainties of real-life clinical } \\
\text { practice (i.e., avoids "hindsight bias")? } \\
\text { ๑ } 1\end{array}$ & $\begin{array}{l}\text { 4. What is the impact of hindsight } \\
\text { bias in determining the existence of } \\
\text { error? How can we reduce this } \\
\text { impact when determining whether an } \\
\text { error caused an adverse event. } \\
\text { 1. What research methods would be } \\
\text { most useful to measure diagnosis- } \\
\text { related cognitive processes taking } \\
\text { into account evolution of diagnosis } \\
\text { and uncertainty? }\end{array}$ & $\begin{array}{l}\text { 5. How can we best use currently } \\
\text { captured data to identify diagnostic } \\
\text { vulnerabilities? E.g., what are the } \\
\text { appropriate statistical measures for } \\
\text { diagnostic error using administrative } \\
\text { data sets? E.g. How can we develop } \\
\text { and validate approaches using the } \\
\text { electronic health record to improve } \\
\text { the identification of diagnostic errors } \\
\text { and reduce measurement burden. }\end{array}$ \\
\hline & & $\begin{array}{l}\text { 6. How can we utilize artificial } \\
\text { intelligence or machine learning to } \\
\text { assist with early identification of } \\
\text { errors in diagnosis? }\end{array}$ & \multirow{3}{*}{$\begin{array}{l}\text { 2. How can we accurately } \\
\text { characterize the progression of } \\
\text { diagnoses from presentation to } \\
\text { "final" diagnoses for patient } \\
\text { trajectories? } \\
\text { 9. For the most common conditions } \\
\text { associated with dx-error-related- }\end{array}$} & $\begin{array}{l}\text { 11. To what extent is the definition of } \\
\text { diagnostic error responsible for the } \\
\text { varied rates reported in the literature }\end{array}$ \\
\hline & & \multirow{2}{*}{$\begin{array}{l}\text { 7. How well can a diagnostic trigger } \\
\text { tool perform across diverse hospital } \\
\text { settings, as a means of tracking and } \\
\text { comparing harm rates } \\
\text { (- } 1\end{array}$} & & $\begin{array}{l}\text { 12. How can we discriminate } \\
\text { between true diagnostic error vs. } \\
\text { evolution of patient diagnosis? }\end{array}$ \\
\hline & & & & 13. What are the most ultimate \\
\hline
\end{tabular}

Figure 1 An example of a Trello board. The top board is an example of the state of a Trello board before discussion; all questions appear in the "Undecided" column. The bottom board is an example of the state of a Trello board after discussion; each question is moved to the High, Medium, or Low Priority column.

\section{Expert Selection}

To ensure a broad sample of researchers, we searched for researchers from diverse backgrounds active in a variety of research fields with expertise relevant to diagnostic safety. The search included the following: (1) authors of key diagnostic safety articles in PubMed, Google Scholar, and AHRQ PSNet; (2) NASEM "Improving Diagnosis in Health Care" report committee members, reviewers, and authors frequently cited in the report; ${ }^{15}$ (3) members of research committees of various societies (e.g., Society of General Internal Medicine; Society of Medical Decision Making; Human Factors and Ergonomics Society); and (4) recipients of quality and safety-related grants and awards.

\section{Soliciting Research Questions in Round 1}

We invited the researchers via an email and requested they submit research questions considering the project scope and the five prioritization criteria. If researchers declined participation, we requested a brief reason. If they agreed to participate, they entered the questions as free text in an online questionnaire in Qualtrics Research Suite (Qualtrics, Provo, UT). We asked them to indicate the domain of each question: (1) measuring burden of the problem, (2) identifying contributing factors, (3) developing and testing effectiveness of solutions, and (4) other for miscellaneous questions. 


\section{Expert Meeting}

The list of research questions was initially prioritized at an inperson 1.5-day expert meeting composed of international experts in April 2019 to select high-priority questions (for a list of participants see supplementary material). The group consisted of researchers with expertise in diagnostic safety research as well as related fields such as human factors, informatics, and social sciences. We also included a patient representative and representatives of funders.

Prior to the meeting, the core research group checked the quality of questionnaire responses, removed responses outside the scope and duplicate questions, and merged similar questions. Furthermore, all questions submitted to the "other" domain were recategorized, making this domain obsolete.

During the expert meeting, we conducted a series of prioritization exercises for each of the three domains. The intent was to identify the top 15 research questions in each domain for a total of 45 research questions. We used Trello (Trello by Atlassian, New York City, NY), a free online program for organizing lists and "cards" on a virtual whiteboard (see Fig. 1).

The large group was divided into three equal breakout groups for each of the three domain prioritization exercises (i.e., one each for burden, contributory factors, and interventions) keeping a similar multidisciplinary representation that was present in the larger group. The virtual whiteboards (Fig. 1, top board) initially contained four lists: Undecided (i.e., all of the research questions for that session that needed to be sorted into the other three lists), and High, Medium, and Low Priority. Each group projected their own version of the whiteboard on a screen, discussed each question, and moved the questions from undecided to one of the priority levels (Fig. 1, bottom board). At the end of the breakout, all participants came together for a plenary discussion. At the start of the plenary, responses from the three groups were combined and questions that made the high-priority list in at least two groups automatically moved to the final high-priority list. The entire group then discussed the remaining questions and assigned them to high, medium, or low priority. This process was followed for all three domains.

\section{Stakeholders}

To recognize the important role of non-researcher stakeholders in the field of diagnostic safety, we invited them to weigh the prioritization criteria in order of importance. The weights would affect the final prioritization score. We selected various types of stakeholders based on their engagement, work, or expertise relevant to diagnosis and invited stakeholder representatives from the following categories: risk managers/ patient safety professionals, patient advocates, clinicians, liability insurers, funders, educators, policy makers, health system leaders, developers of decision support systems, and patient safety organizations.
The stakeholders received an email from the study principal investigators with a request to indicate how important they deemed each of the prioritization criteria. If they agreed to participate, they reviewed the prioritization criteria and ranked them in order of importance (" 1 " most important-" 5 " least important) through a short questionnaire in the Qualtrics Research Suite. The weights were determined by calculating the average importance score for each criterion and then dividing the average expected score of 3.0 (i.e., the average expected rank if all criteria were valued the same) by the average score. ${ }^{29}$ Prioritization criteria with a weight $<1.0$ would have a lower contribution in determining the final priority score whereas criteria with a weight $>1.0$ would have a higher contribution in the determination of the priority score.

\section{Final Prioritization in Round 2}

All researchers who submitted questions were invited to score the high-priority questions that resulted from the expert panel using the five predefined prioritization criteria. Researchers indicated whether the question met the criteria (100 points assigned), whether the question did not meet the criteria ( 0 points assigned), or whether they were undecided whether the criteria was met (50 points assigned). Recognizing that researchers would have unique expertise that may not cut across all the diverse areas and disciplines, we requested them to score only the areas they were comfortable with. The scores of the researcher resulted in a priority score for each of the research questions. ${ }^{30}$ To obtain the final weighted Research Prioritization Score (WRPS), the score for each of the criteria was multiplied by the weight assigned to that criterion by the stakeholders.

To obtain insights into the agreement between the researchers who scored the research questions on the prioritization criteria, we used the Average Expert Agreement (AEA) score. ${ }^{30}$ This score represents the average proportion of experts who agreed on the responses for the five prioritization criteria per question and a valuable indicator of the agreement between experts on the importance of the research question.

\section{Ethical Approval}

Ethical approval was reviewed by the Medical Ethics Research Committee of Erasmus Medical Center, Rotterdam, The Netherlands.

\section{RESULTS}

Of the 207 invited researchers, 97 researchers responded (46.9\% response rate). Of this group, 78 researchers from over 10 different countries submitted research questions and 19 declined participation, for lack of time or because they felt they had insufficient expertise in diagnostic safety. The sample represented fields of quality/patient safety, human factors, social science, 


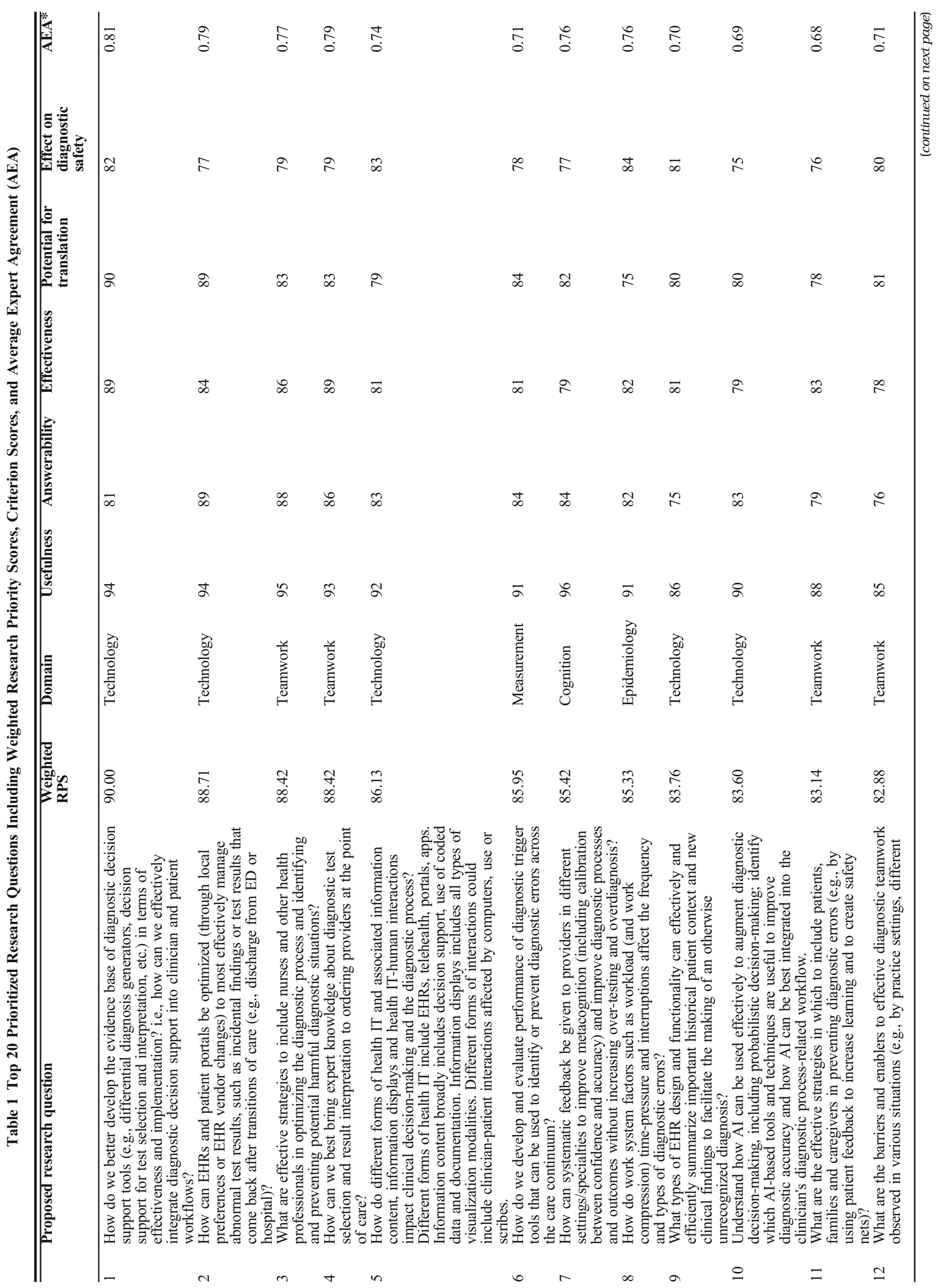




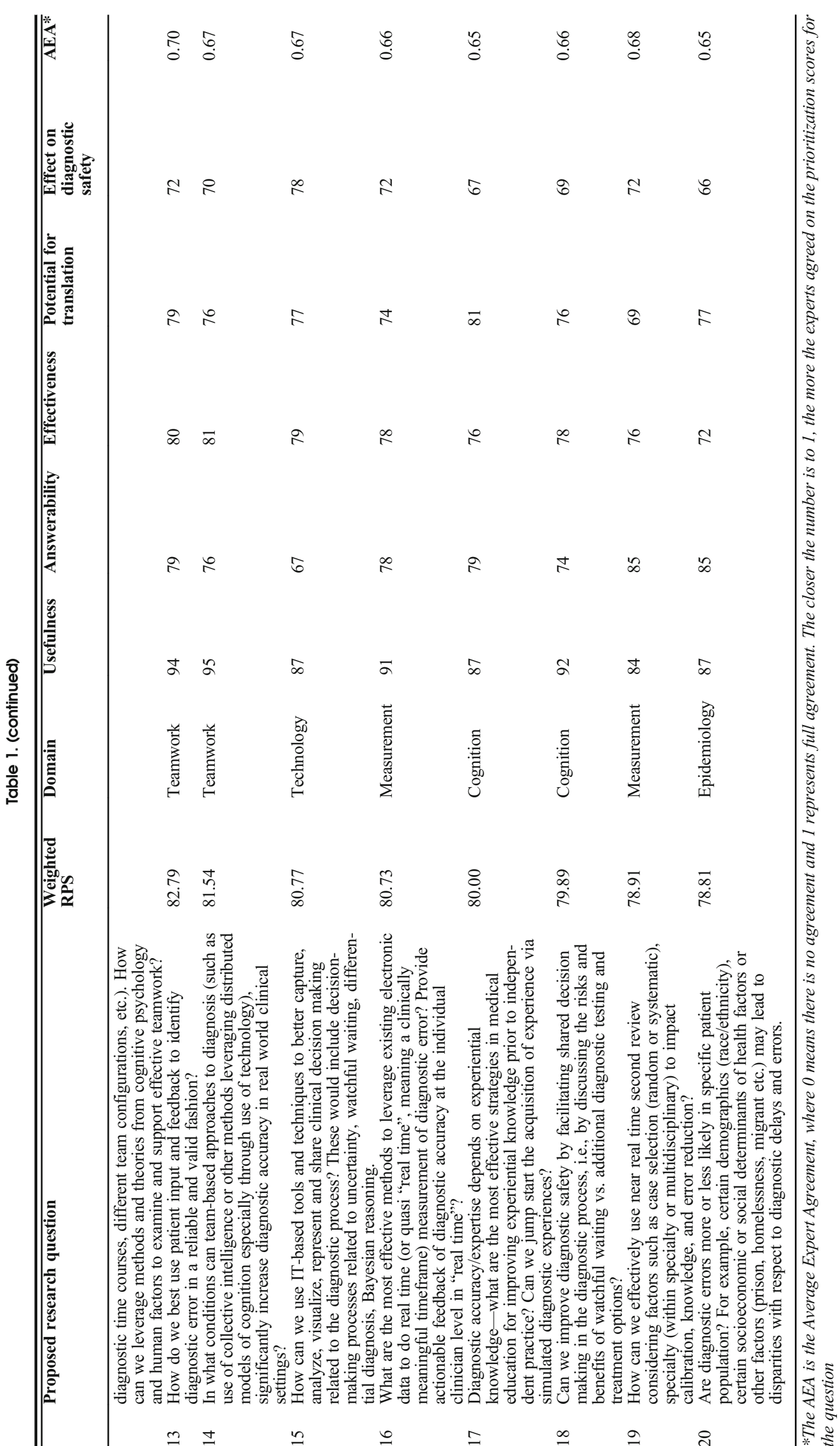


implementation research, health services research, health IT/ informatics, and medical decision making/clinical reasoning.

Researchers submitted 333 research questions in round 1: 77 for measuring burden, 89 for contributing factors, 145 for interventions, and 22 for the other category. Prior to the expert meeting, the core research group consolidated the questions down to 177 questions. This reduction was mainly because of a large number of duplicates and merging of similar questions, but a few questions out of the scope of the project were excluded.

During the expert meeting, participants $(n=21)$ discussed all 177 questions in three different sessions for burden, contributory factors, and interventions respectively. Each session was further divided into three breakout groups that discussed the same questions. The breakout group discussion prioritized 10,10 , and 9 questions immediately to a high-priority list for burden, contributory factors, and interventions respectively. In the plenary group discussions, the remaining questions were discussed resulting in a final high-priority list of 51 questions, 13 questions for measuring burden, 17 questions for contributing factors, and 20 questions for interventions.

Of the 76 invited stakeholders, 43 people responded (response rate $56.6 \%$ ). Of those stakeholders, 42 submitted weights for the prioritization criteria and one person did not consider him/herself a stakeholder for the field. The stakeholders had assigned the weights (1 being a neutral weight). Maximum potential for effect on diagnostic safety $=1.30$; effectiveness $=1.11$; potential for translation $=1.06$; usefulness $=0.95$; and answerability $=0.75$.

Of the 78 invited researchers who submitted questions on round 1, 49 responded (response rate 62.8\%) in the reprioritization exercise in round 2 . On average, the researchers indicated $5.7(11.4 \%)$ questions as outside their expertise. The weighted top 20 questions are listed in Table 1. Specifically, the WRPS are listed as well as each of the prioritization criteria and the AEA score.

\section{DISCUSSION}

Using systematic, transparent, and objective methods that included input from a large group of researchers and stakeholders, we identified a list of top 20 research questions that inform a research agenda that could be supported by several types of funding agencies. Answers to these questions can identify high-risk areas and key underlying causes of diagnostic errors for which promising interventions can be developed and tested. Our findings can guide research funders on development of future requests for proposals as well as encourage researchers to think about specific research ideas, hypothesis, and specific aims within the broader context of the research priorities we identified.

Because we solicited research questions for a 3-5-year horizon, unsurprisingly, 6 of the top 10 research priorities were from the intervention category. Reflecting the current state of unanswered questions ${ }^{31}$ and challenges that have emerged with use of information technology, ${ }^{32,} 33$ technology-related questions emerged prominently in the top 10 . These questions focused on improving diagnostic decision support systems, improving design and functionality of electronic health records specifically for diagnosis and use of artificial intelligence in the diagnostic process. The higher priority scores to interventions were thus in line with the scope of the project aiming for harm reduction in $3-5$ years. This also reflects the higher weight that stakeholders gave to the evaluation criterion "Maximum Potential for Effect on Diagnostic Safety." Strategies to engage patients in the diagnostic process and reducing error also emerged prominently and so did research on teamwork, consistent with other areas of patient safety. ${ }^{34,} 35$

Emphasis on teamwork is especially relevant given that diagnosis is a team sport that involves several members including the frontline clinicians, patients, nurses, laboratory/ pathology, and radiology.

Our project used a collaborative interdisciplinary approach to develop questions rather than individual disciplines coming up with their own priorities for reducing diagnostic error. This approach is consistent with the team sport nature of diagnosis and likely led to different results than what may have been seen if each member group had worked in silos. For instance, addressing test ordering and selection problems involves not just frontline clinicians such as emergency, hospital, and general adult and pediatric medicine but also laboratorians and radiologists. Each should have input for research questions. As a result, while several testing-related questions that crossed disciplines emerged in the top 20, more specific and individual research priorities, such as within the laboratory or radiology fields, did not. Another advantage of using this disciplineagnostic approach was emergence of novel ideas pertinent to several aspects of medicine, for instance how to deliver feedback to clinicians about their diagnostic performance. A collaborative approach across researchers from multiple disciplines thus enhanced the quality of the research questions.

Questions related to measurement of burden and contributing factors did not emerge to the top, even though this could be considered as a required concurrent or initial step to examining the effectiveness of interventions. For instance, several systemic factors need to be addressed in order to make changes including legal and regulatory issues, malpractice-related concerns, and defensive medicine, developing a safety culture and overcoming frontline implementation challenges to solve quality and safety problems. All of these "thorny" issues are fundamentally essential to success of any interventions. ${ }^{36,37}$ Additionally, while many technology-related questions emerged, IT solutions have been challenging to implement in the current environment because of several sociotechnical factors including poor software usability. ${ }^{17}$ Many of these longer term factors important to make changes in clinical practice are not reflected in our research priorities. Addressing both the complex nature of human cognitive processes and the deeply rooted systemic factors influencing the diagnostic process is essential long-term research and implementation priorities. ${ }^{38}$ 
Our study strengths include representation of a diverse group of international researchers from a variety of disciplines and use of systematic methods. Involving a large number of researchers from a variety of societies and disciplines limits the potential of personal biases to influence the outcome. We were able to address all three of the large domains previously identified as foundational to advance the field. ${ }^{14}$

Our limitations include a modest initial response rate, which could likely be due to otherwise busy researchers being asked to commit time to respond in absence of specific incentives. Developing and rating research questions is a time-consuming task with high cognitive workload, which affects response rates. Response rates for the CHNRI method typically vary between 30 and $70 \%$ for both researchers and stakeholders; ${ }^{25}$ thus, the response rates are comparable to other studies using this method. The response rate for the final prioritization exercise was much higher, reflecting an engaged group of researchers. Researchers could have been disinclined to reveal their best research questions. However, we did not witness this and in fact witnessed instances in our expert meeting where researchers advocated for their questions to be included in the priority list.

We also walked a fine line between including a broad group of participants while simultaneously ensuring sufficient expertise. Some invited participants from "outside" the research field of diagnostic safety declined participation because they felt they did not have enough knowledge to identify or evaluate research priorities in diagnostic safety. Lastly, our questions may not be fully representative. For instance, while we received input from at least 10 different countries, this may not be representative of research priorities worldwide. We included a patient representative but did not invite research questions from patient groups. We also did not delve deeper into discipline-specific research areas (e.g., lab, radiology) or how to develop capacity and a cadre of researchers to answer these research questions. Given the recent funding momentum in the field, ${ }^{23,} 39$ a new federal inter-agency task force on diagnostic safety ${ }^{40}$ and more disciplines expressing high interest in diagnosis, these areas will be developed in due course.

In conclusion, the study identified the top short-term research priorities for advancing diagnostic safety that would be useful to both researchers and funders interested in reducing diagnostic error. Priorities broadly included addressing systems, teams, and patient engagement to support diagnosis and serve as a foundation to improve diagnostic safety and reduce preventable diagnostic harm in the near term.

Supplementary Information The online version contains supplementary material available at https://doi.org/10.1007/s11606-02006428-3.

Acknowledgments: The authors wish to thank Melissa Feldman, for her assistance in organizing the expert meeting. Furthermore, they wish to thank the researchers who participated in this study for their time and expertise in submitting and scoring research questions, and the stakeholders for their valuable input in ranking the prioritization criteria.
Corresponding Author: Laura Zwaan, PhD; Erasmus Medical Center Rotterdam, Institute of Medical Education Research Rotterdam, Rotterdam, The Netherlands (e-mail: l.zwaan@erasmusmc.nl).

Funding This project was funded by the Gordon and Betty Moore foundation. Dr. Zwaan is additionally supported by a VENI grant from the Dutch National Scientific Organization (NWO; 45116032). Drs. Meyer and Singh are additionally supported in part by the Houston VA HSR\&D Center for Innovations in Quality, Effectiveness and Safety (CIN13-413). Dr. Meyer is also supported by a VA HSR\&D Career Development Award (CDA-17-167). Dr. Singh is additionally supported by the VA HSR\&D Service (CRE17-127 and the Presidential Early Career Award for Scientists and Engineers USA 14-274), the VA National Center for Patient Safety, and the Agency for Healthcare Research and Quality (R01HS27363). Dr. El-Kareh is additionally supported by the National Institute of General Medical Sciences (R01GM118609).

Data Availability The datasets of the current study will be available from the corresponding author on reasonable request.

\section{Compliance with Ethical Standards:}

Conflict of Interest: The authors declare that they do not have a conflict of interest.

Open Access This article is licensed under a Creative Commons Attribution 4.0 International License, which permits use, sharing, adaptation, distribution and reproduction in any medium or format, as long as you give appropriate credit to the original author(s) and the source, provide a link to the Creative Commons licence, and indicate if changes were made. The images or other third party material in this article are included in the article's Creative Commons licence, unless indicated otherwise in a credit line to the material. If material is not included in the article's Creative Commons licence and your intended use is not permitted by statutory regulation or exceeds the permitted use, you will need to obtain permission directly from the copyright holder. To view a copy of this licence, visit http://creativecommons. org/licenses/by/4.0/.

\section{REFERENCES}

1. Bates DW, Singh H. Two Decades Since To Err Is Human: An Assessment Of Progress And Emerging Priorities In Patient Safety. Health Aff (Millwood). 2018;37(11):1736-1743. https://doi.org/10.1377/hlthaff.2018.0738.

2. Singh H, Meyer AND, Thomas EJ. The frequency of diagnostic errors in outpatient care: estimations from three large observational studies involving US adult populations. BMJ Qual Saf. 2014;23(9):727-731. https://doi.org/10.1136/bmjqs-2013-002627.

3. Raffel KE, Kantor MA, Barish P, et al. Prevalence and Characterization of Diagnostic Error among 7-day All-Cause Hospital Medicine Readmissions: A Retrospective Cohort Study. BMJ Qual Saf. Published online 2020.

4. Zwaan L, Thijs A, Wagner C, van der Wal G, Timmermans DRM. Relating faults in diagnostic reasoning with diagnostic errors and patient harm. Acad Med. 2012;87(2):149-156. https://doi.org/10.1097/ACM.0b013e31823f7le6.

5. Gunderson CG, Bilan VP, Holleck JL, et al. Prevalence of harmful diagnostic errors in hospitalised adults: a systematic review and metaanalysis. BMJ Qual Saf. Published online April 8, 2020. https://doi.org/ 10.1136/bmjqs-2019-010822.

6. Henriksen KS, Dymek C, Harrison MI, Brady PJ, Arnold SB. Challenges and opportunities from the Agency for Healthcare Research and Quality (AHRQ) research summit on improving diagnosis: a proceedings review. Diagnosis. 2017;4(2):57-66. https://doi.org/10.1515/dx-2017-0016.

7. Schiff GD, Martin SA, Eidelman DH, et al. Ten Principles for More Conservative, Care-Full Diagnosis. Ann Intern Med. 2018;169(9):643. https://doi.org/10.7326/M18-1468.

8. Graber M, Gordon R, Franklin N. Reducing Diagnostic Errors in Medicine: What's the Goal? Acad Med. 2002;77(10):981-992.

9. Zwaan L, Singh $\mathbf{H}$. The challenges in defining and measuring diagnostic error. Diagnosis. 2015;2(2):97-103. 
10. Singh H, Schiff GD, Graber ML, Onakpoya I, Thompson MJ. The global burden of diagnostic errors in primary care. BMJ Qual Saf. 2017;26(6):484-494. https://doi.org/10.1136/bmjqs-2016-005401.

11. Zwaan L, Hautz WE. Bridging the gap between uncertainty, confidence and diagnostic accuracy: calibration is key. 2019;28(5):352-355

12. Schiff GD, Hasan O, Kim S, et al. Diagnostic error in medicine: analysis of 583 physician-reported errors. Arch Intern Med. 2009;169(20):18811887. https://doi.org/10.1001/archinternmed.2009.333.

13. Zwaan L, Singh H. Diagnostic error in hospitals: finding forests not just the big trees. BMJ Qual Saf. Published online August 4, 2020. https:// doi.org/10.1136/bmjqs-2020-011099.

14. Zwaan L, Schiff GD, Singh $\mathbf{H}$. Advancing the research agenda for diagnostic error reduction. BMJ Qual Saf. 2013;22(Suppl 2):ii52-ii57.

15. National Academies of Sciences E. Improving Diagnosis in Health Care.; 2015. https://doi.org/10.17226/21794.

16. Henriksen $\mathbf{K}, \mathbf{B r a d y} \mathbf{J}$. The pursuit of better diagnostic performance: a human factors perspective. BMJ Qual Saf. 2013;22(Suppl 2):ii1-ii5. https://doi.org/10.1136/bmjqs-2013-001827.

17. Sittig DF, Singh H. A New Socio-technical Model for Studying Health Information Technology in Complex Adaptive Healthcare Systems. Qual Saf Health Care. 2010;19(Suppl 3):i68-i74. https://doi.org/10.1136/ qshc.2010.042085.

18. Patient Safety Strategies Targeted at Diagnostic ErrorsA Systematic Review | Annals of Internal Medicine | American College of Physicians. Accessed January 27, 2020. https://annals.org/aim/fullarticle/1656430/patient-safety-strategies-targeted-diagnostic-errors-systematic-review.

19. Chopra V, Harrod M, Winter S, et al. Focused Ethnography of Diagnosis in Academic Medical Centers. J Hosp Med. 2018;13(10):668-672. https://doi.org/10.12788/jhm.2966.

20. Sarkar U, Simchowitz B, Bonacum D, et al. A Qualitative Analysis of Physician Perspectives on Missed and Delayed Outpatient Diagnosis: The Focus on System-Related Factors. Jt Comm J Qual Patient Saf. 2014;40(10):AP1. https://doi.org/10.1016/S1553-7250(14)40059-X.

21. Roadmap for Research to Improve Diagnosis. Society to Improve Diagnosis in Medicine. Accessed January 22, 2020. https://www. improvediagnosis.org/roadmap/.

22. Durning SJ, Trowbridge RL, Schuwirth L. Clinical Reasoning and Diagnostic Error: A Call to Merge Two Worlds to Improve Patient Care. Acad Med. 2020;Publish Ahead of Print. https://doi.org/10.1097/ACM. 0000000000003041.

23. The Moore Foundation invests $\$ 85$ million to improve diagnostic performance. Accessed September 27, 2019. https://www.moore.org/ article-detail?newsUrlName=the-moore-foundation-invests-\$85-millionto-improve-diagnostic-performance.

24. Child Health and Nutrition Research Initiative (CHNRI) approach to research priority setting. Published August 21, 2019. Accessed August 21, 2019. /prioritysetting/blog/child-health-and-nutrition-research-initiative-chnri-approach-research-priority-setting.

25. Rudan I, Yoshida S, Chan KY, et al. Setting health research priorities using the CHNRI method: VII. A review of the first 50 applications of the CHNRI method. J Glob Health. 2017;7(1):011004. https://doi.org/10. 7189/jogh.07.011004.

26. Nagata JM, Ferguson BJ, Ross DA. Minding the Gap: Setting Research Priorities Related to HIV Testing, Treatment, and Service Delivery Among
Adolescents. J Adolesc Health. 2018;63(2):131-132. https://doi.org/10. 1016/j.jadohealth.2018.03.009.

27. Tomlinson M, Swartz L, Officer A, Chan KY, Rudan I, Saxena S. Research priorities for health of people with disabilities: an expert opinion exercise. Lancet. 2009;374(9704):1857-1862. https://doi.org/10.1016/ S0140-6736(09)61910-3.

28. Rudan I, Gibson JL, Ameratunga S, et al. Setting Priorities in Global Child Health Research Investments: Guidelines for Implementation of the CHNRI Method. Croat Med J. 2008;49(6):720-733. https://doi.org/10. 3325/cmj.2008.49.720.

29. Yoshida S, Wazny K, Cousens S, Chan KY. Setting health research priorities using the CHNRI method: III. Involving stakeholders. J Glob Health. 6(1). https://doi.org/10.7189/jogh.06.010303.

30. Irvine C, Armstrong A, Nagata JM, et al. Setting Global Research Priorities in Pediatric and Adolescent HIV Using the Child Health and Nutrition Research Initiative (CHNRI) Methodology. J Acquir Immune Defic Syndr 1999. 2018;78(1):S3-S9. https://doi.org/10.1097/QAI. 0000000000001742 .

31. El-Kareh R, Hasan O, Schiff GD. Use of health information technology to reduce diagnostic errors. BMJ Qual Saf. 2013;22 Suppl 2:ii40-ii51. https://doi.org/10.1136/bmjqs-2013-001884.

32. Schiff GD, Bates DW. Can Electronic Clinical Documentation Help Prevent Diagnostic Errors? N Engl J Med. 2010;362(12):1066-1069. https://doi.org/10.1056/NEJMp0911734.

33. Graber ML, Byrne C, Johnston D. The impact of electronic health records on diagnosis. Diagn Berl Ger. 2017;4(4):211-223. https://doi org/10.1515/dx-2017-0012.

34. Update: Patient Engagement in Safety | AHRQ Patient Safety Network Accessed September 10, 2019. https://psnet.ahrq.gov/perspectives/ perspective / 263 / Update-Patient-Engagement-in-Safety? q=patient+engagement.

35. Salas E, Rosen MA. Building high reliability teams: progress and some reflections on teamwork training. BMJ Qual Saf. 2013;22(5):369-373. https://doi.org/10.1136/bmjqs-2013-002015.

36. Braithwaite J, Marks D, Taylor N. Harnessing implementation science to improve care quality and patient safety: a systematic review of targeted literature. Int J Qual Health Care. 2014;26(3):321-329. https://doi.org/ 10.1093/intqhe/mzu047.

37. Sullivan HR, Schweikart SJ. Are Current Tort Liability Doctrines Adequate for Addressing Injury Caused by AI? AMA J Ethics. 2019;21(2):160-166. https://doi.org/10.1001/amajethics.2019.160.

38. Norman G. Research in clinical reasoning: past history and current trends. Med Educ. 2005;39(4):418-427.

39. Grants to Enable Diagnostic Excellence. Accessed January 22, 2020. http://www.ahrq.gov/patient-safety/diagnostic-error-grants/index. html.

40. Diagnostic Safety and Quality. Accessed August 10, 2020. https://www. ahrq.gov/topics/diagnostic-safety-and-quality.html.

Publisher's Note Springer Nature remains neutral with regard to jurisdictional claims in published maps and institutional affiliations. 